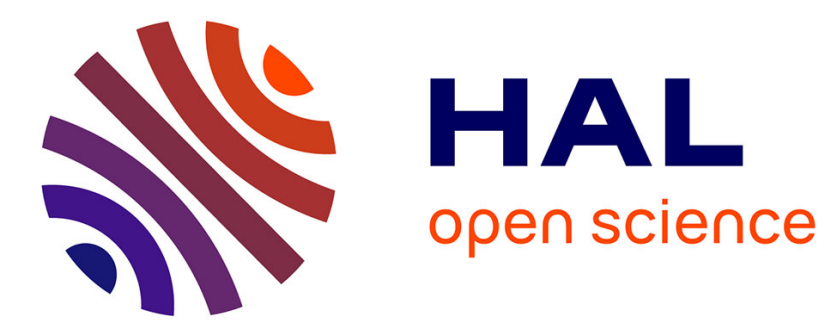

\title{
Positron Spectroscopy of Defects in Semiconductors
}

P. Hautojärvi

\section{To cite this version:}

P. Hautojärvi. Positron Spectroscopy of Defects in Semiconductors. Journal de Physique IV Proceedings, 1995, 05 (C1), pp.C1-3-C1-14. 10.1051/jp4:1995101 . jpa-00253537

\section{HAL Id: jpa-00253537 https://hal.science/jpa-00253537}

Submitted on 1 Jan 1995

HAL is a multi-disciplinary open access archive for the deposit and dissemination of scientific research documents, whether they are published or not. The documents may come from teaching and research institutions in France or abroad, or from public or private research centers.
L'archive ouverte pluridisciplinaire HAL, est destinée au dépôt et à la diffusion de documents scientifiques de niveau recherche, publiés ou non, émanant des établissements d'enseignement et de recherche français ou étrangers, des laboratoires publics ou privés. 


\title{
Positron Spectroscopy of Defects in Semiconductors
}

\author{
P. Hautojärvi
}

Laboratory of Physics, Helsinki University of Technology, FIN-02150 Espoo, Finland

\begin{abstract}
At a vacant lattice cell positron-ion repulsion is reduced leading to positron trapping. This causes observable changes in annihilation characteristics: the positron lifetime increases and the positron-electron momentum distribution narrows. Positron trapping in semiconductors is analogous to carrier capture. Due to the long-range Coulomb interaction, the charge state of a vacancy has a strong effect on positron trapping. The lattice relaxation due to a charge-state transition of a vacancy is also seen in the positron lifetime. Thus ionization levels of vacancy defects can be determined.

Applications are shown on native vacancies in GaAs. Positrons reveal $\mathrm{As}$ and $\mathrm{Ga}$ vacancies in bulk crystals. The As vacancies have negative, neutral and positive charge states in the upper part of the band gap, whereas Ga vacancies are negative. The EL2 defect has a vacancy in its metastable state. A vacancy is also seen in the deep ground state of the DX center in AlGaAs. Positron results thus support the vacancy-interstitial model for the structure of the EL2 and DX centers.
\end{abstract}

\section{INTRODUCTION}

Atomic defects in semiconductors are electrically active and play a significant role in the electrical and optical properties of the materials. The defects induce localized electron levels which act as trapping, recombination and scattering centers for free carriers. The localized levels can be detected by various spectroscopies. However, the identification of the atomic structure of the defects has turned out to be difficult.

Positron annihilation as a defect-spectroscopic technique has three advantages. First, it has a specific sensitivity to vacancy-type defects which makes their identification straightforward. Second, the positron spectroscopy is strongly supported by theory, as the experimental signal from electron-positron annihilation can be theoretically calculated. Third, positron annihilation can be applied to any material independent of its doping and conductivity.

The positron spectroscopy of defects in metallic materials was developed in the 1970's [1-4]. In semiconductors, the positron-defect interaction is more complicated and a sufficient level of understanding has been reached only recently [4,5]. Around 1980 the technique of low energy positron beams was developed [6]. This opened the avenue of positron studies of near-surface regions, epitaxial layers and interfaces. 


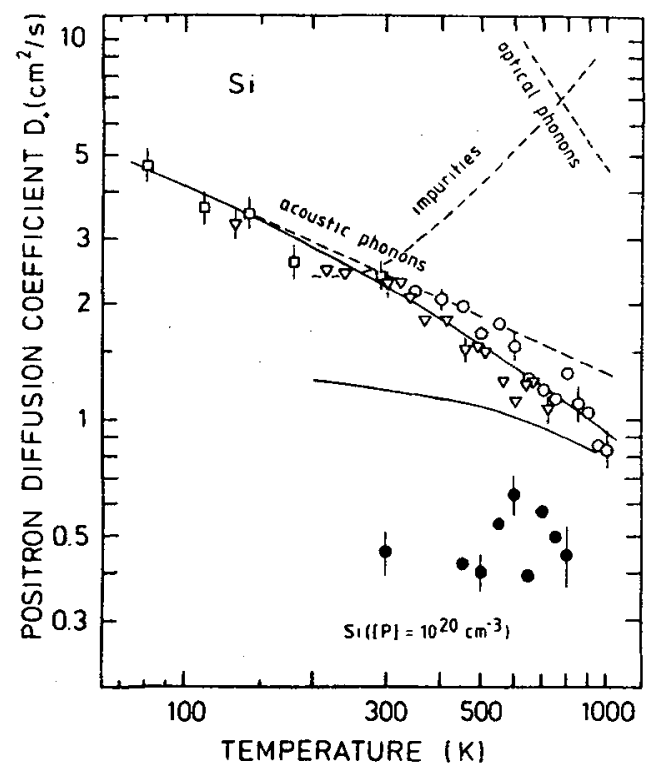

(a)

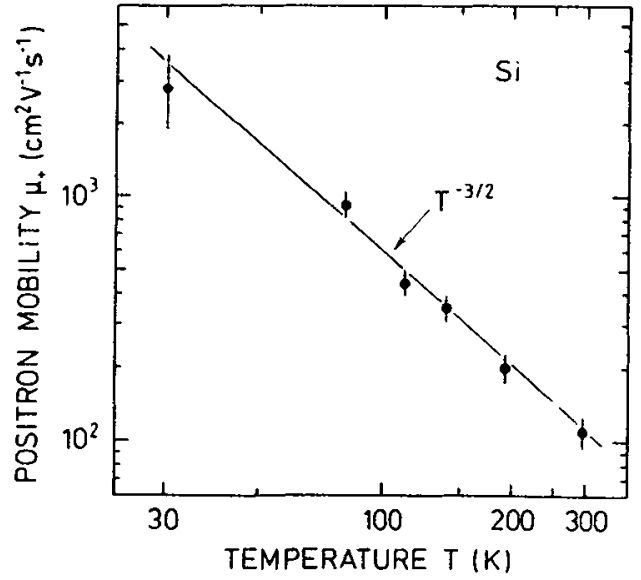

(b)

Fig. 1. Positron diffusion and mobility in Si. (a) Positron diffusion is limited mainly by acoustic phonons. Optical phonons contribute above $300 \mathrm{~K}$. From Soininen et al. [7]. (b) Below $300 \mathrm{~K}$ the mobility follows $T^{-3 / 2}$ dependence due to acoustic phonons. From Mäkinen et al. [8].

In this paper we first discuss the positron states and interactions in semiconductors, and briefly comment on the experimental aspects. The last part of the paper deals with applications to defect spectroscopy using GaAs materials as examples. Results on native vacancies as well as on the metastable centers EL2 and DX are summarized.

\section{POSITRON STATES IN SEMICONDUCTORS}

\subsection{Free positrons}

Energetic positrons in solids thermalize within a few picoseconds. In semiconductors the mean lifetime of free positrons, $\tau_{\mathrm{b}}$, is around $250 \mathrm{ps}$. The motion and trapping of thermal positrons are in many respects analogous to those of free carriers except for the "heavy" positron effective mass $\mathrm{m}^{*} \approx 1.5 \mathrm{~m}_{\mathrm{o}}$. The positron diffusion coefficient at $300 \mathrm{~K}$ is about $2 \mathrm{~cm}^{2} \mathrm{~s}^{-1}[7,8]$. The diffusion is limited mainly by acoustic phonons leading to a $\mathrm{T}^{-1 / 2}$ temperature dependence (figure 1a). Positrons feel electric fields and have a mobility of $100 \mathrm{~cm}^{-2} \mathrm{~V}^{-1} \mathrm{~s}^{-1}$ at $300 \mathrm{~K}$ with a T-3/2 dependence (figure $1 \mathrm{~b}$ ).

\subsection{Vacancies as positron traps}

The Bloch-state positron wave function is squeezed into the interstitial regions between atoms by the positron-ion repulsion. Thus an open-volume defect like a vacant lattice cell is an attractive center where positrons get trapped. Figure 2 shows free and trapped positron wave functions [9]. 


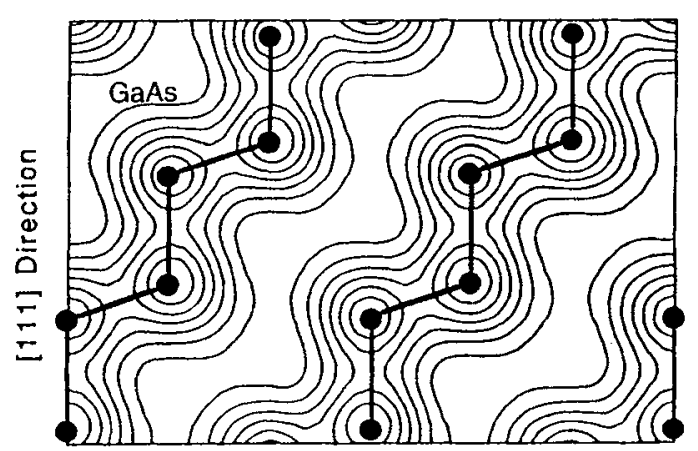

[112] Direction

(a)

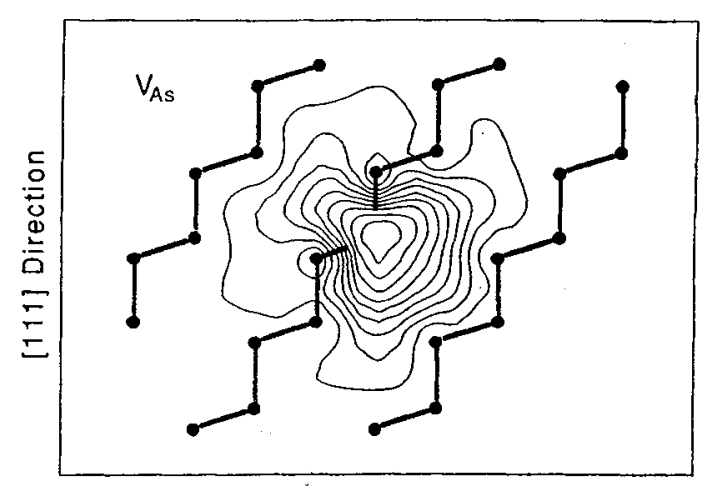

[112] Direction

(b)

Fig. 2. Positron wave functions in GaAs. (a) Free positron in the Bloch state, (b) trapped positron in an As vacancy. From Puska and Corbel [9].

Due to the different electronic environment the annihilation characteristics of trapped positrons are different from those of free positrons. The reduced electron density increases the positron lifetime at a vacancy by $10-20 \%$ [10]. In addition, the momentum distribution of the annihilating pairs becomes narrower. Even the change in the charge state of a vacancy may lead to observable effects in the annihilation characteristics. Examples are the As vacancy in GaAs [11,12] and the vacancy-phosphorous pair in Si [13].

\subsection{Negative ions as positron traps}

Positrons can get localized also at Rydberg states around negative ions [14]. Since there is no open volume, the annihilation characteristics are the same as for free positrons in the bulk. The negative ions are shallow traps with a binding energy of 10-100 meV. Their effect is best seen at low temperature as traps which compete with vacancies.

\section{ON EXPERIMENTAL TECHNIQUES}

\section{1. Positron source}

Positrons are obtained from radioactive isotopes. Their mean energy is a few hundred keV with a stopping range of 10-100 $\mu \mathrm{m}$. Thus they give information of the crystal bulk.For the near-surface region and thin film studies, a low-energy positron beam is needed. Positrons from radioisotopes are first slowed down in a separate moderator. Thermalized positrons close to the moderator surface are spontaneously emitted into vacuum. They form a beam which is accelerated and guided to the sample. In an experiment monoenergetic positrons are implanted at different depths of the sample by varying the beam energy E typically from 0 to $40 \mathrm{keV}$. In Si this energy range scans the depths from 0 to $4 \mu \mathrm{m}$. The positron stopping profile is, however, rather wide which limits the depth resolution of the technique. For a review on positron beams and their applications to semiconductors see Refs. $[6,15]$. 


\subsection{Positron lifetime}

Positron lifetime is the most powerful experimental technique (see the review of Eldrup [1]). A positron lifetime spectrum contains various lifetime components due to different positron states in a sample. In practice, the lifetime spectra are analyzed with 1-3 exponential decay components. The positron trapping rates at various defects can be calculated from the lifetimes $\tau_{i}$ and intensities $I_{i}[1,2]$. The longer lifetimes $\left(\tau_{2}, \tau_{3}\right)$ are defect-specific corresponding to annihilations at lattice defects.

The decomposition may be difficult if the positron trapping fraction is small. The average lifetime $\tau_{\mathrm{av}}=\Sigma \mathrm{I}_{\mathrm{i}} \tau_{\mathrm{i}}$ is a good and reliable parameter to monitor even small changes. When $\tau_{\mathrm{av}}>\tau_{\mathrm{b}}$ vacancy-type defects are present. For details of the lifetime analysis see Ref. [4].

\subsection{Doppler broadening}

A detailed description of the experimental technique can be found in Refs. [1,2,4]. The motion of the annihilating pair causes a Doppler shift which is seen as a broadening of the $511 \mathrm{keV}$ radiation line. The line shape is usually characterized by two parameters. The $S$ parameter is the relative fraction of counts in the central region of the $511 \mathrm{keV}$ peak. It monitors mainly the changes in the momentum distribution of the valence electrons. The $\mathrm{W}$ parameter is the relative fraction of counts in the wing region of the line where only core electron annihilations can contribute.

The changes in the S-parameter due to positron trapping at vacancies is only $2 \%$. Often the wing parameter $\mathrm{W}$ can show bigger changes. The parameter $\mathrm{R}=\Delta \mathrm{S} / \Delta \mathrm{W}$ and the $\mathrm{S}-\mathrm{W}$ plot can give defect-specific information, although the annihilation line is still a superposition of free and trapped positrons.

The Doppler broadening is easy to use. The disadvantage is that the $511 \mathrm{keV}$ line is a superposition of all positron states existing in the sample.

\section{DEFECT IDENTIFICATION}

The increase of the trapped positron lifetime reflects the open volume of a defect. The ratio of the defect and bulk lifetimes $\tau_{\mathrm{d}} / \tau_{\mathrm{b}}$ is 1.1-1.2 for a monovacancy, 1.3-1.4 for a divacancy, and a value $>1.5$ corresponds to a vacancy cluster or void $[9,10]$. It seems that at least in $\mathrm{Si}$ and GaAs the S-parameter can also be used to characterize the open volume. $S_{d} / S_{b}$ is 1.02-1.03 for a monovacancy, 1.03-1.04 for a divacancy and $>1.5$ for a larger vacancy cluster or void.

Information on the defect charge can be obtained from the positron trapping rate. Positive defects do not trap positrons. A neutral defect shows a temperature-independent trapping rate whereas the trapping rate at a negative defect increases at low temperature [16].

The identification of the chemical surroundings or the sublattice of a defect is important especially in compund semiconductors. Very often the sublattice is identified by indirect arguments based e.g. on the defect charge predicted by theory [11,12]. The momentum density of the annihilation radiation of a trapped positron contains information on the atoms surrounding the defect. This can be monitored by complete 2D-ACAR experiments or simply by Doppler broadening measurements [17]. 


\section{DEFECT CONCENTRATION}

The positron trapping rate $\kappa$ is proportional to the defect concentration $C, \kappa=\mu \mathrm{C}$. To determine defect concentrations from experimental trapping rates we need to know the trapping coefficient $\mu$. The trapping coefficient in semiconductors depends strongly on the defect type, defect charge, and host material. We have collected recent experimental values for Si and III-V compounds in Table I. The uncertainties are due to difficulties in knowing the underlying defect concentrations. The estimates are based on electron irradiation (e--irr), electron-paramagnetic- resonance (EPR), infrared absorption (IR) or Hall measurements. For negative defects $\mu=10^{15}-10^{16}$ at. $\mathrm{s}^{-1}$ at $300 \mathrm{~K}$ and $10^{16}-10^{17}$ at. $\mathrm{s}^{-1}$ at $20 \mathrm{~K}$. For neutral vacancies $\mu$ is assumed to be independent of temperature and around $10^{15} \mathrm{at}^{-1} \mathrm{~s}^{-1}$ [16]. These numbers mean that concentrations above $10^{15} \mathrm{~cm}^{-3}(\sim 0.1 \mathrm{ppm})$ can be detected.

We should keep in mind that the trapping coefficient is still a relatively unknown parameter and considerable revisions of the values presented in Table I are possible. Further experiments are needed where positron annihilation and conventional defect analysis techniques are correlated.

TABLE I. Some recent experimental values of positron trapping coefficients $\mu$ in semiconductors. Also the complementary method is mentioned.

\begin{tabular}{|c|c|c|c|c|}
\hline $\begin{array}{l}\text { Material } \\
\text { Defect }\end{array}$ & $\begin{array}{l}\mu\left(10^{15}\right. \\
300 K\end{array}$ & $\begin{array}{ll}-1) \\
20 K\end{array}$ & Method & Reference \\
\hline $\begin{array}{l}S i \\
V^{-} \\
(\mathrm{V}-\mathrm{P})^{\circ} \\
(\mathrm{V}-\mathrm{P})^{-} \\
\mathrm{V}_{2}{ }^{\circ} \\
\mathrm{V}_{2}^{-}\end{array}$ & $\begin{array}{l}1 \pm 0.5 \\
2 \pm 1 \\
1 \\
3.5\end{array}$ & $\begin{array}{l}>100 \\
20 \pm 10 \\
40\end{array}$ & $\begin{array}{l}\mathrm{e}^{-} \text {irr/guess } \\
\mathrm{e}^{-} \text {irr/[P] } \\
\mathrm{e}^{-} \text {irr/[P] } \\
\mathrm{e}^{-\mathrm{irr} / \mathrm{EPR}} \\
\mathrm{e}^{-} \text {irr//EPR }\end{array}$ & $\begin{array}{l}\text { Mäkinen et al. [18] } \\
\text { Mäkinen et al. [13] } \\
\text { Mäkinen et al. [13] } \\
\text { Mascher et al. [19] } \\
\text { Mascher et al. [19] }\end{array}$ \\
\hline $\begin{array}{l}\text { GaAs } \\
\mathrm{V}^{3-} \mathrm{Ga} \\
\mathrm{V}_{\mathrm{As}} \\
\mathrm{Ga}^{2-} \text { As } \\
\mathrm{EL}^{\text {As }}\end{array}$ & $\begin{array}{l}1.4 \pm 0.2 \\
1.4 \pm 0.2 \\
3 \pm 1 \\
--\end{array}$ & $\begin{array}{l}13 \pm 2 \\
13 \pm 2 \\
29 \pm 5\end{array}$ & $\begin{array}{l}\text { e-irr/compensation } \\
\text { the value for } \mathrm{V}_{\mathrm{Ga}}{ }^{3-} \\
\mathrm{e}^{-} \text {irr/detrapping } \\
\text { IR abs }\end{array}$ & $\begin{array}{ll}\text { Corbel et al. } & {[14]} \\
\text { Saarinen et al. } & {[27]} \\
\text { Corbel et al. } & {[14]} \\
\text { LeBerre } & {[20]}\end{array}$ \\
\hline $\begin{array}{l}G a P \\
V_{\mathrm{P}^{-}}\end{array}$ & $1.9 \pm 0.5$ & & Hall & Krause et al. [21] \\
\hline $\begin{array}{l}\text { AlGaAs } \\
\text { DX }\end{array}$ & $2 \pm 1$ & & Hall & Mäkinen et al: [22] \\
\hline $\begin{array}{l}\boldsymbol{A l G a S b} \\
\text { DX }\end{array}$ & $1 \pm 0.5$ & & Hail & Krause et al. \\
\hline
\end{tabular}




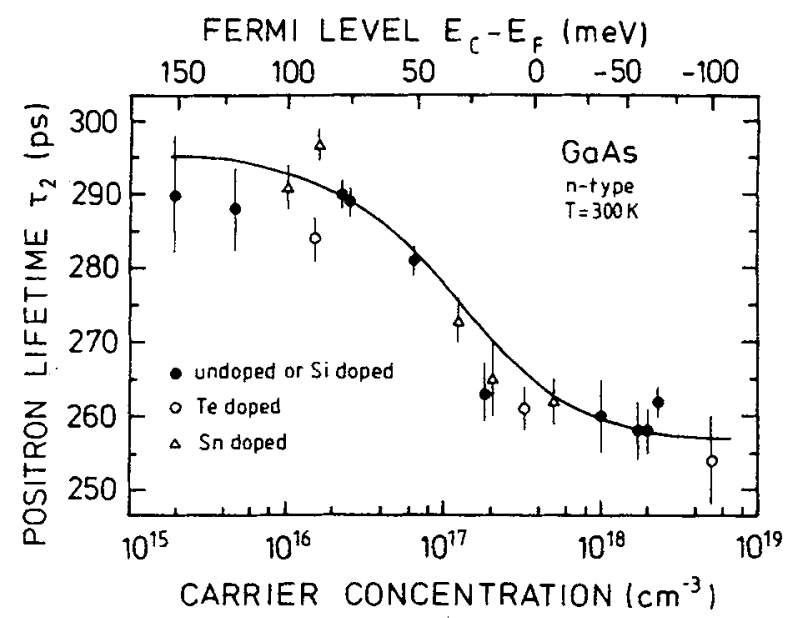

FIG. 3. Positron lifetime $\tau_{2}$ at a native vacancy as a function of the carrier concentration in $n-G a A s$ at $300 \mathrm{~K}$. The vacancy is identified to As vacancy. From Saarinen et al. [12].

\section{NATIVE As AND Ga VACANCIES IN GaAs}

\subsection{As vacancies and their charge states in n-type GaAs}

The free positron lifetime in GaAs is $231 \mathrm{ps}$ at $300 \mathrm{~K}$. In n-type bulk crystals, independent of the growth technique, the positron lifetime $\tau_{\mathrm{av}}$ is always above this value indicating the presence of native vacancies. The lifetime of positrons at vacancies is clearly correlated with the carrier concentration of the sample (figure 3) [11,12]. A defect lifetime of $295 \mathrm{ps}$ is obtained at low n-type doping, whereas in heavily n-type material it is replaced by a lifetime of 257 ps. When compared to theoretical calculations, both of these lifetimes correspond to positrons annihilating while trapped at defects of the size of a monovacancy [9].

When the measurement temperature of a $\mathrm{n}-\mathrm{GaAs}$ crystal is varied, the lifetime of trapped positrons changes reversibly from 257 ps to 295 ps. Furthermore, the transition temperature depends on the carrier concentration of the sample, and it changes from roughly $80 \mathrm{~K}$ to about $550 \mathrm{~K}$, when the room temperature carrier concentration is increased from $\mathrm{n}=1.9 \times 10^{15} \mathrm{~cm}^{-3}$ to $\mathrm{n}=1.8 \times 10^{17} \mathrm{~cm}^{-3}$ [12]. This suggests strongly that the lifetime transition $257 \mathrm{ps} \rightarrow 295 \mathrm{ps}$ is not a pure temperature effect, but rather that it is controlled by the Fermi level in the energy gap. The lifetime transition $257 \mathrm{ps} \rightarrow 295 \mathrm{ps}$ occurs at a Fermi level position of $30 \mathrm{meV}$ below the conduction band edge $E_{c}$ for a wide range of carrier concentrations. Also, the solid line in figure 3 is the Fermi function with $\mathrm{E}_{\mathrm{F}}=\mathrm{E}_{\mathrm{c}}-30 \mathrm{meV}$.

The trapping rate to the 295 ps state of the native vacancy vanishes when the Fermi level decreases further (the sample temperature increases). Also this process is controlled by the Fermi level position and the corresponding ionization level is at $E_{c}-140 \mathrm{meV}$ [12].

According to calculations, As vacancies have ionization levels in the upper part of the gap whereas Ga vacancies have levels in the lower half of the gap $[24,25]$. Therefore the native vacancy in $\mathrm{n}$-GaAs is identified as the As vacancy. Below $\mathrm{E}_{\mathrm{c}}-140 \mathrm{meV}$ the charge is positive $\left(\mathrm{V}_{\mathrm{As}}{ }^{+}\right)$as the 


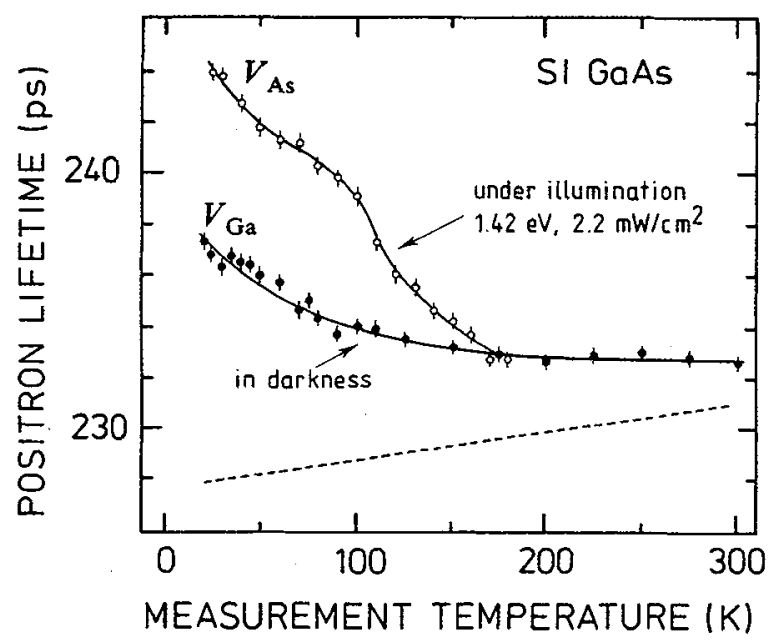

Fig. 4. Positron lifetime $\tau_{a v}$ as a function of temperature in semi-insulating GaAs. Ga vacancies are seen in darkness and As vacancies are revealed under illumination. From Saarinen et al. [27].

positron trapping vanishes. The transitions $\mathrm{V}_{\mathrm{As}}{ }^{+} \rightarrow \mathrm{V}_{\mathrm{As}}{ }^{\circ}$ and $\mathrm{V}_{\mathrm{As}}{ }^{\circ} \rightarrow \mathrm{V}_{\mathrm{As}}{ }^{-1}$ occur at $\mathrm{E}_{\mathrm{C}}-140 \mathrm{meV}$ and $\mathrm{E}_{\mathrm{c}}-30 \mathrm{meV}$, respectively.

Further, the lifetime change from 257 ps to 295 ps indicates that a large lattice relaxation is present in the transition $\mathrm{V}_{\mathrm{As}}{ }^{-1} \rightarrow \mathrm{V}_{\mathrm{As}}{ }^{\circ}$. This is confirmed by later Car-Parrinello calculations [25] and by $2 \mathrm{D}$-ACAR measurements [26].

It is important to bear in mind that the positron lifetime does not indicate whether the As vacancy is isolated or bound to an impurity or other defect. If the As vacancy is a part of a defect complex the charge state seen by positrons is the total charge of the complex.

The $\mathrm{V}_{\text {As }}$ concentrations can be easily calculated from the experimental trapping rates. The trapping coefficient depends both on the charge state and on temperature. With $\mu=10^{15}$ at s$^{-1}$ (Table I) we find that the As vacancy concentration in $\mathrm{n}-\mathrm{GaAs}$ is around $10^{16}-10^{17} \mathrm{~cm}^{-3}$ which is high compared to that of any other defects in GaAs.

\subsection{Ga and As vacancies in semi-insulating GaAs}

In semi-insulating (SI) material the Fermi level is at the mid-gap position. The As vacancies are positive and do not trap positrons, whereas the Ga vacancies are negative (2- or 3-) and constitute potential traps for positrons.

At $300 \mathrm{~K}$ the lifetime $\tau_{\mathrm{av}}$ in SI-GaAs is $0-3$ ps above the free positron value $[11,12]$. The experimental sensitivity can be improved by lowering the sample temperature. The trapping coefficient $\mu$ for a negative vacancy increases by almost an order of magnitude at $20 \mathrm{~K}$ as is seen in Table I. The low-temperature measurements in darkness show the presence of a negative vacancy (figure 4) which has been identified as the $\mathrm{Ga}$ vacancy on the basis of its charge [27]. 
Measurements under illumination with $1.4 \mathrm{eV}$ photons reveal another type of vacancy (figure 4) which has a negative charge state above $\mathrm{E}_{\mathrm{c}}-50 \mathrm{meV}$ [27]. This vacancy is identified as the As vacancy which has trapped electrons due to photoexcitation. The concentrations of both $\mathrm{Ga}$ and As vacancies are between $10^{15}$ and $10^{16} \mathrm{~cm}^{-3}$. These values are an order-of-magnitude less than the As vacancy concentrations in $\mathrm{n}$-GaAs.

\subsection{Vacancies in epitaxial GaAs layers}

When state-of-the-art homoepitaxial GaAs/GaAs layers are grown, experiments with a low-energy positron beam reveal no defects. This means that the vacancy concentrations must be below $10^{15} \mathrm{~cm}^{-3}$. On the other hand, heteroepitaxial $\mathrm{GaAs} / \mathrm{Si}$ layers may contain vacancies and vacancy clusters in the range of $10^{16}-10^{17} \mathrm{~cm}^{-3}$ [28]. If the growth temperature is low, around $200^{\circ} \mathrm{C}$, vacancy concentrations above $10^{18} \mathrm{~cm}^{-3}$ are observed [29].

\section{METASTABLE DEFECTS}

\subsection{EL2 defect in GaAs}

The EL2 (electron level 2) defect is a native midgap donor in GaAs [30]. It plays a key role in the growth of undoped semi-insulating GaAs material by compensating residual acceptors. The interesting property of EL2 is its metastability. The EL2 level disappears after illumination below 100 $\mathrm{K}$. The defect is transformed into the metastable state EL2*. It reappears into the stable state by annealing above $120 \mathrm{~K}$. The metastable state $\mathrm{EL} 2 *$ does not give any optical or electrical signal.

An important discovery by positron annihilation is that when EL2 is in the metastable state positrons are trapped at a vacancy defect $[31,32]$. This 'metastable vacancy' disappears as EL $2 *$ is converted to the stable state (see figure 5a). The lifetime at the metastable state is $245 \mathrm{ps,} \mathrm{which} \mathrm{is}$ between the bulk lifetime $230 \mathrm{ps}$ and the Ga vacancy lifetime $260 \mathrm{ps}$. The lifetime ratio is $\tau_{\mathrm{EL} 2} / \tau_{\mathrm{b}}=$ 1.07 , which is smaller than that of the Ga vacancy, $\tau_{\mathrm{VGa}} / \tau_{\mathrm{b}}=1.13$.

Saarinen et al. [32] have correlated the optical properties of EL2 to positron trapping. Figure 5a shows that during the conversion EL2 $\rightarrow$ EL2* the infrared absorption disappears and the positron trapping at EL2* appears. From the kinetic curves it is possible to deduce the optical cross section for the EL2 conversion. Figure 5b shows that the cross sections determined either by IR absorption or by positron trapping show perfect agreement both in absolute magnitude and photon energy dependence. This confirms that the positron is indeed trapped by EL2*. LeBerre et al. [20] have correlated the EL2 concentration determined by IR absorption to positron trapping and found the trapping coefficient of $\mathrm{EL} 2 *$ at $20 \mathrm{~K}$ to be $\mu_{\mathrm{EL} 2 *}=2.9 \times 10^{16}$ at.s $^{-1}$. 


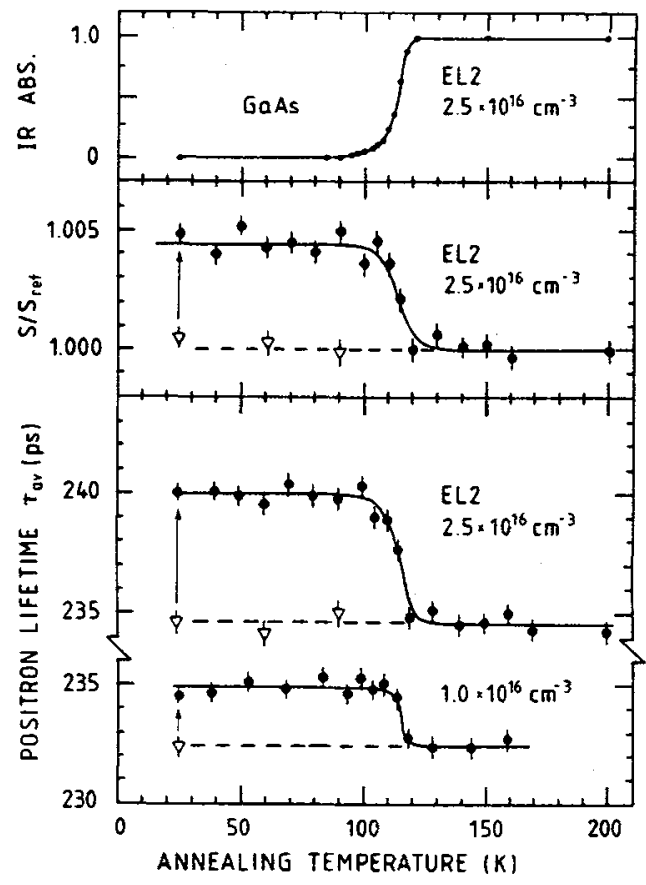

(a)

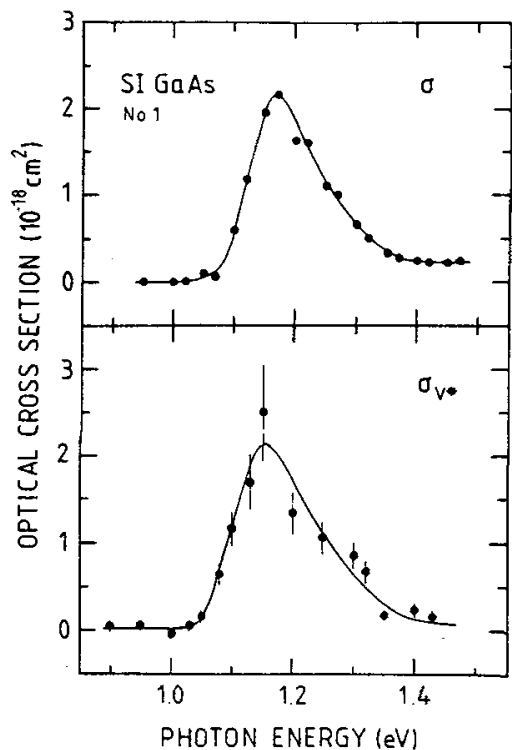

(b)

Fig. 5. (a) Normalized IR absorption and positron annihilation characteristics as functions of annealing temperature after illumination with $1.2 \mathrm{eV}$ photons in S1-GaAs with two EL2 concentrations. From Krause et al. [31]. (b) Optical cross section for the creation of the metastable state of EL2 as a function of photon energy. The upper curve is from IR absorption and the lower one from positron trapping. From Saarinen et al. [32].

\subsection{DX center in $\mathrm{Al}_{\mathbf{x}} \mathrm{Ga}_{1-\mathrm{x}} \mathrm{As}$}

Group IV impurity atoms like Si and Sn are normally shallow donors in III-V compounds. In n-type $\mathrm{Al}_{\mathrm{x}} \mathrm{Ga}_{1-\mathrm{x}} \mathrm{As}$ and related ternary alloys they may also create deep donor levels, i.e. they do not donate electrons to the conduction band at room temperature. These levels are called DX (unidentified donor) centers [33]. The DX level has metastable properties: After illumination at low temperature the DX level disappears leading to persistent photoconductivity, i.e. to a persistent increase in the number of conduction electrons. The DX level reappears after annealing above $100 \mathrm{~K}$.

Positron annihilation finds a vacancy defect associated with the DX center. Figure 6a shows the Doppler results measured in various $\mathrm{Al}_{x} \mathrm{Ga}_{1-\mathrm{x}}$ As layers by Mäkinen et al. [22] using a positron beam. In undoped layers the core annihilation parameter $\mathrm{W}$ shows a smooth behavior with the AlAs mole fraction $\mathrm{x}$. However, in $\mathrm{Si}$ and $\mathrm{Sn}$ doped layers, the $\mathrm{W}$ values are clearly below the level of the undoped layers. This is attributed to the presence of vacancies: a trapped positron at a vacancy has less overlap with core electrons than a free positron. Illumination at $20 \mathrm{~K}$ brings the $\mathrm{W}$ parameter of the doped layers to the level of the undoped layers indicating the removal of the positron trapping. The 


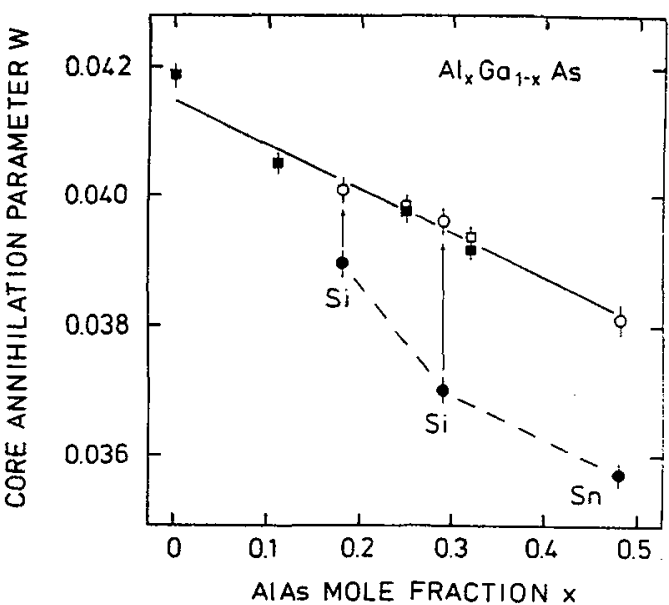

(a)

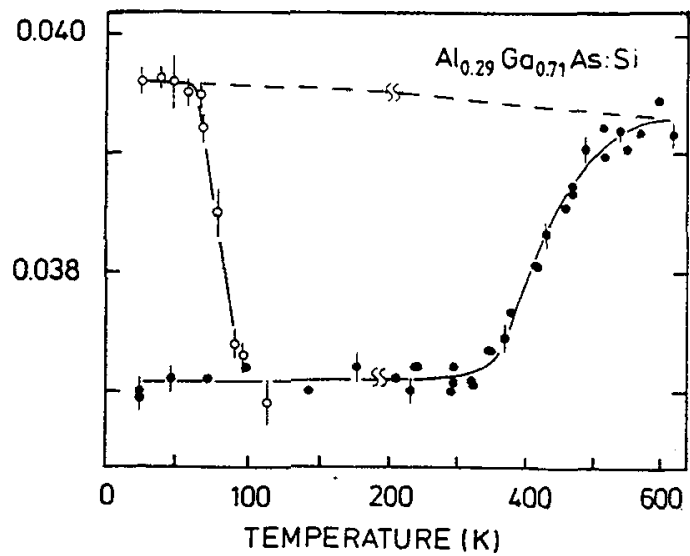

(b)

FIG. 6. (a) The core annihilation parameter $W$ in $A l_{x} G a_{1-x}$ As layers. Illumination by $1.3 \mathrm{eV}$ photons at $25 \mathrm{~K}$ lifts the $W$ values of the Si and Sn doped layers to the reference line of the undoped layers.

(b) The W parameter as a function of temperature in a Si-doped layer. Filled circles: measured in the dark. Open circles: measured in the dark after exposure to $1.3 \mathrm{eV}$ photons at $25 \mathrm{~K}$. From Mäkinen et al. [22].

disappearance of the positron trapping is metastable, as shown in figure $6 \mathrm{~b}$. After annealing above 100 $\mathrm{K}$ the value of $\mathrm{W}$ drops down again in the Si-doped layers. The vacancy trapping is removed also at higher temperatures above $300 \mathrm{~K}$ because the DX center is thermally ionized. All the positron results indicate that there is a vacancy in the structure of the DX center. When the DX level is occupied positrons see the vacancy. When the DX level is ionized either optically at low temperature or thermally above $300 \mathrm{~K}$ no vacancy is seen.

Saarinen [34] has tried to measure the lifetime at the DX center in Sn-doped $\mathrm{Al}_{\mathrm{x}} \mathrm{Ga}_{1-\mathrm{x}} \mathrm{As}$. Preliminary results give a value of about $245 \mathrm{ps}$. The bulk lifetime $230 \mathrm{ps}$ is the same as in GaAs and the lifetime ratio is $\tau_{\mathrm{DX}} / \tau_{\mathrm{b}}=1.07$. This is about the same as for the EL2* defect and well below the ratio 1.13 for $\mathrm{V}_{\mathrm{Ga}}$. Krause-Rehberg et al. [23] have performed lifetime experiments at the DX center in Te-doped bulk $\mathrm{Al}_{\mathrm{X}} \mathrm{Ga}_{1-\mathrm{x}} \mathrm{Sb}$. They find the value $\tau_{\mathrm{DX}} / \tau_{\mathrm{b}}=295 \mathrm{ps} / 270 \mathrm{ps}=1.09$ confirming that the open volume of the DX center is relatively small.

In summary, the positron experiments demonstrate that the metastable state EL $2 *$ as well as the stable state of the DX center contain a vacancy defect. The open volume in both centers is about the same and smaller than that of a monovacancy. These findings form an important step towards the understanding of the atomic structure and metastability of these centers.

\subsection{The microscopic model of EL2 and DX}

The EL2 center is associated with the As antisite defect $\mathrm{As}_{\mathrm{Ga}}$. This is a double donor because an As atom with five valence electrons has replaced a $\mathrm{Ga}$ atom with three valence electrons. 
According to calculations [35,36] a donor atom ( $\mathrm{As}_{\mathrm{Ga}}$ in $\mathrm{GaAs}$, Si and $\mathrm{Sn}$ in $\mathrm{Al}_{\mathrm{x}} \mathrm{Ga}_{1-\mathrm{x}} \mathrm{As}$ etc.) has two positions in the lattice, a stable and a metastable one, with almost the same energy. One position is the normal lattice site with $\mathrm{sp}^{3}$ bonding whereas the other is the interstitial position in the $<111>$ direction with $\mathrm{sp}^{2}$ bonding leaving the Ga site vacant. In the case of EL2, the unrelaxed configuration is the stable one, whereas in the case of DX the relaxed configuration is the stable one:

$$
\begin{gathered}
\mathrm{EL} 2 \rightarrow \mathrm{EL} 2^{*}: \quad \mathrm{As}_{\mathrm{Ga}} \rightarrow \mathrm{As}_{\mathrm{i}}+\mathrm{V}_{\mathrm{Ga}} \\
\mathrm{DX} \rightarrow \mathrm{D}^{*}: \mathrm{D}_{\mathrm{i}}+\mathrm{V}_{\mathrm{Ga}} \rightarrow \mathrm{D}_{\mathrm{Ga}}
\end{gathered}
$$

Positrons get trapped at $\mathrm{V}_{\mathrm{Ga}}$. Because of the interstitial atom in the immediate vicinity, the open volume experienced by positrons is reduced and the lifetime increase is less than that for an isolated $\mathrm{V}_{\mathrm{Ga}}$.

Laasonen et al. [37] have studied the positron localization at $\mathrm{V}_{\mathrm{Ga}}$ and $\mathrm{EL} 2 *$. If the lifetime at $\mathrm{V}_{\mathrm{Ga}}$ is adjusted to $260 \mathrm{ps}$ by choosing the positron effective mass $\mathrm{m}^{*}=1.5 \mathrm{~m}_{\mathrm{o}}$ then the positron at the EL2* defect have a lifetime of $247 \mathrm{ps,} \mathrm{in} \mathrm{good} \mathrm{agreement} \mathrm{with} \mathrm{experiments.}$

\section{CONCLUSIONS}

The basic physics of positron interactions in semiconductors is well understood. Consequently, positron annihilation has become a real spectroscopic tool for studyingvacancy-type defects. Monovacancies, divacancies and bigger vacancy clusters can be easily identified by the positron lifetime or Doppler broadening of the annihilation line. In addition, negative ions (acceptors) can be observed as shallow traps. The momentum density of the annihilation radiation from trapped positrons contains potential information on the chemical surroundings and sublattice of defects.

\section{REFERENCES}

[1] Eldrup M., these proceedings

[2] Hautojärvi P., Ed., Positrons in Solids (Topics in Current Physics vol. 12) (Springer, Berlin 1979).

[3] Dupasquier A. and Mills P., Eds., Positron Spectroscopy of Solids (North Holland, Amsterdam) in press.

[4] Hautojärvi P. and Corbel C., in Ref. [3].

[5] Corbel C. and Hautojärvi P., in Ref. [3].

[6] Schultz P.J. and Lynn K.G., Rev. Mod. Phys. 60 (1988) 701-779.

[7] Soininen E., Mäkinen J., Beyer D., and Hautojärvi P., Phys. Rev. B 46 (1992) 13104-13118.

[8] Mäkinen J., Corbel C., Hautojärvi P., and Mathiot D., Phys. Rev. B 43 (1991) 12114-12117.

[9] Puska M.J. and Corbel C., Phys. Rev. B 38 (1988) 9874-9880.

[10] Puska M.J., Mäkinen S., Manninen M., and Nieminen R.M., Phys. Rev. B 39 (1989) 7666-7679.

[11] Corbel C., Stucky M., Hautojärvi P., Saarinen K., and Moser P., Phys. Rev. B 38 (1988) 8192-8208.

[12] Saarinen K., Hautojärvi P., Lanki P., and Corbel C., Phys. Rev. B 44 (1991) 10585-10600.

[13] Mäkinen J., Hautojärvi P., and Corbel C., J. Phys.: Condens. Matter 4 81992) 5137-5154.

[14] Corbel C., Pierre F., Saarinen K., Hautojärvi P., and Moser P., Phys. Rev. B 45 (1988) 3386-3399.

[15] Lynn K.G. et al., in Ref. [3], see also these Proceedings. 
[16] Puska M.J., Corbel C., and Nieminen R.M., Phys. Rev. B 41 (1990) 9980-9993.

[17] Alatalo M., Kauppinen H, Saarinen K., Puska M.J., Hautojärvi P., and Nieminen R.M., Phys. Rev. $B$, in print.

[18] Mäkinen J., Corbel C., Hautojärvi P., Moser P., and Pierre F., Phys. Rev. B 39 (1989) 10162-10173.

[19] Mascher P., Dannefaer S., and Kerr D., Phys. Rev, B 40 (1989) 11764-11711.

[20] LeBerre C., Ph.D. thesis, Univ. of Paris 1964; see also Brozel M., these Proceedings.

[21] Krause R., Polity A., Siegel W., and Kühnel G., Semicond. Sci. Technol. 8 (1993) 280-2XX.

[22] Mäkinen J., Laine T., Saarinen K., Hautojärvi P., Corbel C., Airaksinen V.M., and Gibart P., Phys. Rev. Lett. 71 (1993) 3154-3157.

[23] Krause-Rehberg R., Drost Th., Polity A., Roos G., Pensl G., Voln D., Mayer B.K., Bischopink G., and Benz K.W., Phys. Rev. B 48 (1993) 11723-11726.

[24] Baroff G.A., and Schlüter M., Phys. Rev. Lett. 55 (1985) 1327-1330.

[25] Laasonen K., Nieminen R.M., and Puska M.J., Phys. Rev. B 45 (1992) 4122-4130.

[26] Ambigapathy R., Manuel A.A., Hautojärvi P., Saarinen K., and Corbel C., Phys. Rev. B. see also Manuel A.A. et al., these Proceedings.

[27] Saarinen K., Kuisma S., Hautojärvi P., Corbel C., and LeBerre C., Phys. Rev. Lett. 70 (1993) 2794-2797.

[28] Soininen E., Mäkinen J., Hautojärvi P., Corbel C., Freundlich A., and Grenet J.C., Phys. Rev. B 46 (1992) 12394-12401.

[29] Hautojärvi P., Mäkinen J., Palko S., Saarinen K., Corbel C., and Liszkay L., Mat. Sci. Eng. B22 (1993) 16-22.

[30] Martin G.M., Makram-Ebeid S., in "Deep Centers in Semiconductors", ed. by Panthelides S.T. (Gordon and Breach, New York, 1986).

[31] Krause R., Saarinen K., Hautojärvi P., Polity A., Gärtner G., and Corbel C., Phys. Rev. Lett. 65 (1990) 3329-32.

[32] Saarinen K., Kuisma S., Hautojärvi P., Corbel C., and LeBerre C., Phys. Rev. B 49 (1994) 8005-8016.

[33] Mooney P.M., J. Appl. Phys. 67 (1990) R1-R15.

[34] Saarinen K., private communication.

[35] Dabrowski J., and Schefler M., Phys. Rev. Lett. 60 (1988) 2183-2186; Chadi D.J., and Chang K.J., Phys. Rev. Lett. 60 (1988) 2187-2190.

[36] Chadi D.J., and Chang K.J., Phys. Rev. Lett. 61 (1988) 873-876.

[37] Laasonen K., Alatalo M., Puska M.J., and Nieminen R.M., J. Phys.: Condens. Matter 3 (1991) 7217-7224. 